\title{
Development of the SIOPE DIPG network, registry and imaging repository: a collaborative effort to optimize research into a rare and lethal disease
}

Sophie E. M. Veldhuijzen van Zanten ${ }^{1}\left[\right.$ - Joshua Baugh ${ }^{2} \cdot$ Brooklyn Chaney $^{2} \cdot$ Dennis De Jongh $^{3} \cdot$ Esther Sanchez Aliaga $^{4} \cdot$ Frederik Barkhof $^{4,58} \cdot$ Johan Noltes $^{5} \cdot$ Ruben De Wolf $^{5} \cdot$ Jet Van Dijk $^{6}$. Antonio Cannarozzo ${ }^{7}$ - Carin M. Damen-Korbijn ${ }^{8} \cdot$ Jan A. Lieverst $^{8} \cdot$ Niclas Colditz $^{9} \cdot$ Marion Hoffmann $^{9}$. Monika Warmuth-Metz ${ }^{10}$ - Brigitte Bison ${ }^{10}$ • David T. W. Jones ${ }^{11}$ - Dominik Sturm ${ }^{11}$ - Gerrit H. Gielen ${ }^{11}$ •

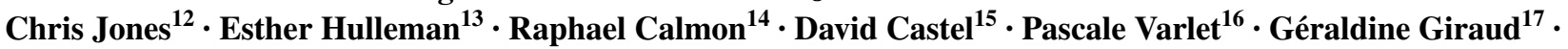
Irene Slavc $^{18}$ - Stefaan Van Gool ${ }^{19}$ • Sandra Jacobs ${ }^{20,21}$ - Filip Jadrijevic-Cvrlje ${ }^{22}$ - David Sumerauer ${ }^{23}$. ${\text { Karsten } \mathrm{Nysom}^{24} \cdot \text { Virve Pentikainen }}^{25} \cdot$ Sanna-Maria Kivivuori $^{26} \cdot$ Pierre Leblond $^{27} \cdot$ Natasha Entz-Werle $^{28}$. Andre O. von Bueren ${ }^{29}$ - Antonis Kattamis ${ }^{30}$ - Darren R. Hargrave ${ }^{31} \cdot$ Péter Hauser $^{32} \cdot$ Miklos Garami $^{32}$. Halldora K. Thorarinsdottir ${ }^{33}$ - Jane Pears ${ }^{34}$ - Lorenza Gandola ${ }^{35}$ - Giedre Rutkauskiene ${ }^{36}$ - Geert O. Janssens ${ }^{37}$. Ingrid K. Torsvik ${ }^{38}$ • Marta Perek-Polnik ${ }^{39} \cdot$ Maria J. Gil-da-Costa ${ }^{40}$ - Olga Zheludkova $^{41} \cdot$ Liudmila Shats $^{42}$. Ladislav Deak ${ }^{43}$ - Lidija Kitanovski ${ }^{44}$ - Ofelia $\mathrm{Cruz}^{45}$ • Andres Morales La Madrid ${ }^{45}$ - Stefan Holm ${ }^{46}$. Nicolas Gerber ${ }^{47} \cdot$ Rejin Kebudi $^{48} \cdot$ Richard Grundy $^{49} \cdot$ Enrique Lopez-Aguilar $^{50} \cdot$ Marta Zapata-Tarres $^{51}$. John Emmerik ${ }^{52}$ - Tim Hayden ${ }^{53} \cdot$ Simon Bailey $^{54} \cdot$ Veronica Biassoni $^{55}$ - Maura Massimino ${ }^{55}$. Jacques Grill $^{17}$ - William P. Vandertop ${ }^{56}$. Gertjan J. L. Kaspers ${ }^{1,57} \cdot$ Maryam Fouladi $^{2}$ Christof M. Kramm9 ${ }^{\text {. }}$ Dannis G. van Vuurden ${ }^{1} \cdot$ on behalf of the members of the SIOPE DIPG Network

Received: 25 July 2016 / Accepted: 25 December 2016 / Published online: 21 January 2017

(C) The Author(s) 2017. This article is published with open access at Springerlink.com

Electronic supplementary material The online version of this article (doi:10.1007/s11060-016-2363-y) contains supplementary material, which is available to authorized users.

Sophie E. M. Veldhuijzen van Zanten

s.veldhuijzen@vumc.nl

1 Department of Paediatrics, Division of Oncology/ Haematology, VU University Medical Center, Amsterdam, The Netherlands

2 Department of Pediatrics, Cancer and Blood Diseases Institute, Cincinnati Children's Hospital Medical Center, Cincinnati, USA

3 2TCI B.V., Breda, The Netherlands

4 Department of Radiology and Nuclear Medicine, VU University Medical Center, Amsterdam, The Netherlands

5 KPMG, Amstelveen, The Netherlands

6 Yellow Research B.V., Amsterdam, The Netherlands

7 Trasferimento Tecnologico (TTO), Fondazione IRCCS Istituto Nazionale dei Tumori, Milano, Italy

8 Dutch Childhood Oncology Group (DCOG), The Hague, The Netherlands

9 Division of Pediatric Hematology and Oncology, Department of Child and Adolescent Health, University of Göttingen, Göttingen, Germany
Abstract Diffuse intrinsic pontine glioma (DIPG) is a rare and deadly childhood malignancy. After 40 years of mostly single-center, often non-randomized trials with variable

10 Division of Pediatric Neurooncology, German Cancer Research Center (DKFZ) Heidelberg, Heidelberg, Germany

11 Institute of Neuropathology, University of Bonn Medical Center, Bonn, Germany

12 Divisions of Molecular Pathology and Cancer Therapeutics, The Institute of Cancer Research, Sutton, UK

13 Neuro-oncology Research Group, VU University Medical Center, Amsterdam, The Netherlands

14 Université Paris-Descartes, Hôpital Necker-Enfants malades, Paris, France

15 UMR 8203 CNRS, Gustave Roussy, Paris-Saclay University, Villejuif, France

16 Service de Neuropathologie, Hôpital Sainte-Anne, Paris, France

17 Institut Gustave Roussy, Villejuif, France

18 Department of Pediatrics and Adolescent Medicine, Medical University of Vienna, Vienna, Austria

19 Immuno-oncology Centre Köln, Cologne, Germany

20 Department of Pediatric Hematology and Oncology, University Hospitals Leuven, Leuven, Belgium 
patient inclusions, there has been no improvement in survival. It is therefore time for international collaboration in DIPG research, to provide new hope for children, parents and medical professionals fighting DIPG. In a first step towards collaboration, in 2011, a network of biologists and clinicians working in the field of DIPG was established within the European Society for Paediatric Oncology (SIOPE) Brain Tumour Group: the SIOPE DIPG Network. By bringing together biomedical professionals and parents as patient representatives, several collaborative DIPG-related projects have been realized. With help from experts in the fields of information technology, and legal advisors, an international, web-based comprehensive database was developed, The SIOPE DIPG Registry and Imaging Repository, to centrally collect data of DIPG patients. As for April 2016, clinical data as well as MR-scans of 694 patients have been entered into the SIOPE DIPG Registry/Imaging Repository. The median progression free survival is 6.0 months $(95 \%$ Confidence Interval (CI) 5.6-6.4 months) and the median overall survival is 11.0 months (95\% CI 10.5-11.5 months). At two and five years post-diagnosis, 10 and $2 \%$ of patients are alive, respectively. The establishment of the SIOPE DIPG Network and SIOPE DIPG Registry means a paradigm shift towards collaborative research into DIPG. This is seen as an essential first step towards understanding the disease, improving care and (ultimately) cure for children with DIPG.

21 Department of Microbiology and Immunology, KU Leuven, Leuven, Belgium

22 Department of Oncology and Hematology, Children's Hospital Zagreb, Zagreb, Croatia

23 Department of Pediatric Hematology and Oncology, 2nd Faculty of Medicine, University Hospital Motol, Charles University, Prague, Czech Republic

24 Pediatrics and Adolescent Medicine, University Hospital Rigshospitalet, Copenhagen, Denmark

25 Division of Hematology-Oncology and Stem Cell Transplantation, Children's Hospital, Helsinki University Hospital, Helsinki, Finland

26 Helsinki University Hospital (HUH) and HUH Children and Adolescents, Helsinki, Finland

27 Pediatric Oncology Unit, Oscar Lambret Comprehensive Cancer Center, Lille, France

28 Service de Pédiatrie Onco-Hématologie, CHRU Hautepierre Strasbourg, Strasbourg, France

29 Pediatric Oncology and Hematology, Department of Pediatrics, University Hospital of Geneva, Genève, Switzerland

30 First Department of Pediatrics, National and Kapodistrian University of Athens, 'Aghia Sofia' Children's Hospital, Athens, Greece
Keywords Diffuse intrinsic pontine glioma (DIPG) . Collaboration - International research-infrastructure . SIOPE DIPG network · SIOPE DIPG registry

\section{Introduction}

Diffuse intrinsic pontine glioma (DIPG) is a pediatric brain cancer for which there is no curative treatment yet. Despite multiple clinical trials studying (combinations of) cytotoxic chemotherapy, including novel agents, the median overall survival of 9 months has not improved over the past decades [1,2]. Although major advances have been accomplished in knowledge on the biological background of the disease by discovery of a high prevalence of specific mutations in genes encoding for histone 3.1 and 3.3, ACVR1 and P53 [3-9], much is yet to be learned on the mechanisms that contribute to treatment resistance. Research on the DIPG patient population, however, is hampered because integrative, large scale clinical, radiological and biological data are lacking.

There are several factors that contribute to the scarcity of data. First, DIPG is an orphan disease with a yearly incidence of 2.32 per $1,000,000$ residents aged 0-20 years [10]. Second, DIPGs are diagnosed clinically, based on typical MR-imaging findings [11], in

31 Paediatric Oncology Unit, Great Ormond Street Hospital (GOSH), London, UK

32 Second Department of Pediatrics, Semmelweis University, Budapest, Hungary

33 Pediatric Hematology-Oncology, The Children's Hospital, Reykjavik, Iceland

34 Department of Paediatric Oncology, Our Lady's Children's Hospital, Crumlin, Dublin, Ireland

35 Pediatric Radiotherapy Unit, Department of Diagnostic Radiology and Radiotherapy, Fondazione IRCCS Istituto Nazionale dei Tumori, Milano, Italy

36 Department of Pediatric Oncology and Hematology, Hospital of Lithuanian University of Health Sciences Kaunas Clinic, Kaunas, Lithuania

37 Department of Radiation Oncology, University Medical Center Utrecht and Princess Maxima Center for Pediatric Oncology, Utrecht, The Netherlands

38 Department of Pediatrics, Division of Oncology/Hematology, Haukeland University Hospital, Mons, Norway

39 Department of Oncology, The Children's Memorial Health Institute, Warsaw, Poland

40 Pediatric Hemathology and Oncology Division, University Hospital S. João Alameda Hernani Monteiro, Porto, Portugal 
combination with a classic triad of neurological symptoms [12]. Biopsy procedures to obtain tumor material have long been considered dangerous and not contributing to the diagnosis, treatment approach or survival outcome [13]. Fortunately, recent years have seen an emergence of studies that include biopsies, however, the discovery of new mutations have caused an on-going debate about the actual definition of the disease itself [9, 14]. This is exemplified by the recently published new WHO classification of central nervous system tumors, that has reclassified DIPG to the category of WHO grade IV diffuse midline gliomas with histone mutations [15]. Inconsistent definition of DIPG has hampered in- and exclusion or response criteria for clinical trials, which resulted in a great variety of mostly incomparable clinical trials, many of which are single-center, single-arm studies with only few patients enrolled [10].

Collaboration and data sharing are promising strategies for tackling rare diseases, by facilitating uniform and hypothesis-driven research [16]. To overcome the current lack of data and improve the integration, speed, quality, and coherence of research, we aimed to (1) create a DIPG research-infrastructure consortium, and (2) initiate collaborative collection of comprehensive data on DIPG patients. This paper describes the methodology of the set-up of an international research network infrastructure, the SIOPE DIPG Network and SIOPE DIPG Registry, including legal and IT aspects, as well as preliminary patient inclusion data.

41 Department of Neuro-Oncology, Russian Scientific Center of Radiology, Moscow, Russia

42 Department of Children Oncology and Haematology, City Clinical Hospital \#31 of Saint-Petersburg, St.-Petersburg, Russian Federation

43 Department of Pediatric Oncology/ Haematology, Children University Hospital, Kosice, Slovakia

44 Department of Pediatrics, Division of Haematooncology, University Medical Center Ljubljana, Ljubljana, Slovenia

45 Department of Pediatric Hematology and Oncology, Hospital Sant Joan de Déu, Barcelona, Spain

46 Department of Pediatric Hematology and Oncology, Department of Woman and Child Health, Karolinska University Hospital, Stockholm, Sweden

47 Department of Oncology, University Children's Hospital of Zurich, Zurich, Switzerland

48 Cerrahpasa Medical Faculty \& Oncology Institute, Pediatric Hematology-Oncology, Istanbul University, Istanbul, Turkey

49 Children's Brain Tumour Research Centre, The Medical School, University of Nottingham, Nottingham, UK

\section{Materials and methods}

\section{The establishment of a research-infrastructure consortium}

In January 2011, in a DIPG meeting organized by the Semmy Foundation in Amsterdam, the SIOPE DIPG Network was established as a sub-committee of the high-grade glioma (HGG) working group of International Society of Paediatric Oncology Europe (SIOPE). The SIOPE DIPG Network is a collaboration of pediatric oncologists, neurologists, neurosurgeons, radiotherapists, radiologists, pathologists, molecular biologists, psychologists and others motivated to carry out excellent clinical and biological research in the field of DIPG. Initially started as a European network, it has extended to colleagues from all over the world, with participants from Russia, Turkey and Mexico.

The SIOPE DIPG Network is comprised of (i) an executive committee, (ii) a group of scientific advisors, (iii) National Coordinators (NCs) and (iv) members. The Executive Committee (i) manages and controls the DIPG Network, and abides by and enforces the mission and the core values of the Network. Scientific Advisors (ii) are individuals with expertise in areas such as: biostatistics and biometry, medical ethics and health policy, basic science research, translational research, (neuro)psychology, neuroimaging, or other areas not mentioned. Scientific Advisors are consulted to advise the Executive Committee in matters of development and implementation of research protocols including ideas for innovative studies that could

50 Jefatura de Servicio de Oncología, Hospital de Pediatría, Centro Médico Nacional Siglo XXI, Instituto Mexicano del Seguro Social, Distrito Federal, Mexico

51 Division of Pediatric Oncology, Hospital Infantil de Mexico Federico Gomez, Mexico City, Mexico

52 Stichting Semmy, Weesp, The Netherlands

53 The DIPG Collaborative, Cincinnati, USA

54 Great North Childrens Hospital, Victoria Wing, Royal Victoria Infirmary, Newcastle upon Tyne, UK

55 Pediatric Oncology Unit, Fondazione IRCCS Istituto Nazionale dei Tumori, Milano, Italy

56 Neurosurgical Center Amsterdam, Academic Medical Center and VU University Medical Center, Amsterdam, The Netherlands

57 Academy of Princess Máxima Center for Pediatric Oncology, Lundlaan 6, 3584 EA Utrecht, The Netherlands

58 Institutes of Neurology and Healthcare Engineering, UCL, London, UK 


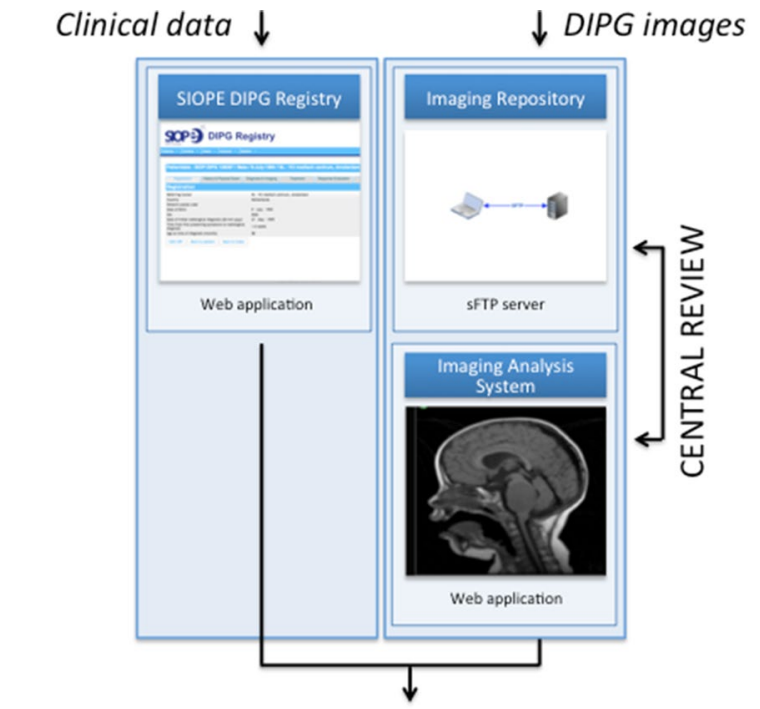

Exhaustivity chèck and quality control of the data*

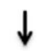

Data storage within the SIOPE DIPG Registry database

Fig. 1 Organizational chart of the SIOPE DIPG registry and imaging repository. For details on the quality control process please see Supplementary Fig. 1

be executed using the Network. NCs (iii) are those DIPG Network members that coordinate collaboration between the SIOPE DIPG Network and biologists and clinicians in their countries. NCs identify and select hospitals and scientific experts in their countries, that are involved in the treatment of DIPG patients and that potentially may join the DIPG Network. DIPG Network members (iv) participate in research projects initiated by the DIPG Network following the principles of Good Clinical Practice. Potential members need to be approved by the Executive Committee before subscription to the DIPG Network. Network members are free to decide on whether they wish to participate in a research project on a case-by-case basis and at their sole discretion.

The mission of the SIOPE DIPG Network is to serve as a research-infrastructure for the design and execution of high quality, international multicenter laboratory and clinical studies, intended to enhance the understanding of DIPG and to improve outcome of patients suffering from DIPG. The mission, aims, core values and structure of the SIOPE DIPG Network are described in the SIOPE DIPG Network Bylaws (see Legal aspects).

\section{Collaborative collection of comprehensive data}

The establishment of a DIPG registry was set as first project of the Network, with the purpose to include clinical, biological and centrally reviewed radiology data of patients with DIPG, both in- and outside clinical trials. The SIOPE DIPG Registry is composed of an online web application and database for clinical data, and an Imaging Repository for radiological data (Fig. 1).

In parallel, an International DIPG Registry was initiated and developed, which includes patient data from the USA, Canada, Australia and New Zealand. To allow for the inclusion of uniform data, standardized electronic Case Report Forms (e-CRFs; Fig. 2) were developed by the SIOPE DIPG Network, in coordination with colleagues from the International DIPG Registry. The online e-CRFs collect
Fig. 2 Screenshot of the SIOPE DIPG Registry showing the electronic case report forms (e-CRFs). The open tab represents the e-CRF for history and physical exam

\section{SOP3. DIPG Registry}

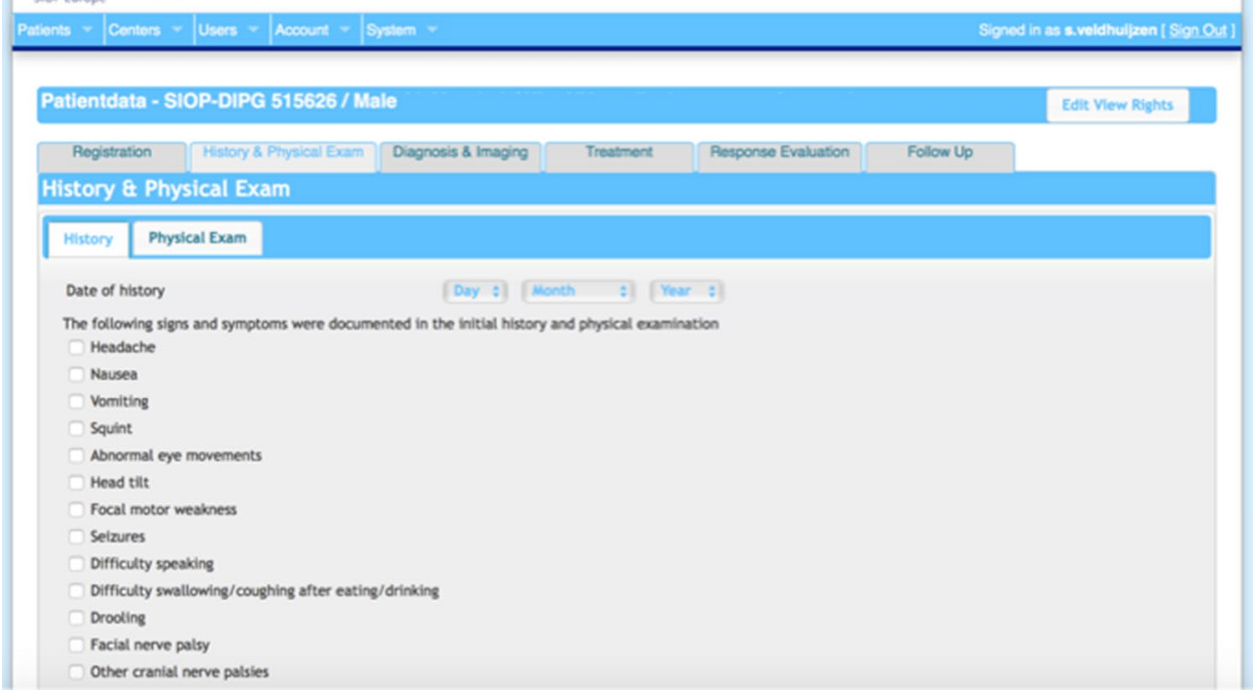


data on demographics, medical history and physical exam at time of diagnosis together with the results from radiological and pathological review by the local hospital, treatment data (including radiotherapy, chemotherapy, surgery and supportive care such as steroids), data on clinical and radiological follow up, and last known status of the patient (see Data Entry Manual; Supplementary material 1).

In parallel to the clinical data, anonymized MRI-scans scans are uploaded via a secure FTP server or sent on CDs. De-identification/ pseudonymization, according to the country's law, is performed either in the referring center, during upload or at the time of receipt. When fully anonymized, these images are uploaded into the SIOPE DIPG Imaging Repository (Fig. 1). Expert neuroradiologists are brought together in a central neuroradiology panel. This panel has access to view assigned images from the Imaging Repository for blinded central review of submitted cases.

\section{Eligibility criteria}

The criteria for patient inclusion in the SIOPE DIPG Registry are: (i) patients with DIPG, or with focal Pontine Glioma (fPG), defined as a T1-weighted hypointense and T2-weighted hyperintense tumor with at least $50 \%$ involvement of the pons (DIPG) or less than 50\% involvement of the pons (fPG) on T2, and as confirmed by expert neuroradiologists via the central radiology review procedure described above (ii) age at diagnosis between 0 and 21 years, and (iii) written informed consent in case of prospective registration. Furthermore, in order to enable validation of the diagnosis following the current guideline, a minimum of diagnostic criteria is required i.e. clinical and radiological data (MRI scans) to be shared in the registry and, if available, pathology data.

\section{Ethical considerations}

The SIOPE DIPG Registry is conducted according to the principles of the Declaration of Helsinki. No personal identifiers, besides date of birth, are included in the e-CRFs. If in a certain country this is not allowed, age at diagnosis is submitted instead. All patients are assigned a unique SIOPE DIPG Registry number. Per member site, a separate list, kept under a special password, connects the DIPG Registry number with the personal identifiers. Access to this list is restricted to a local coordinator at each site.

In most participating countries informed consent is not mandatory for retrospective registration of (mostly deceased) patients. If required a consent form is sent to parents and signed. Prospective registration of living patients requires an informed consent procedure. National coordinators are responsible for the translation of the standardized informed consent to the language of their country. Translated forms will be centrally collected and available to local hospitals upon request. In this procedure, a SIOPE Network member informs parents (and patients), after which he/she provides the Patient Information Form (Supplementary material 2) and requests for informed consent. Parents or patients may reject participation at all times.

\section{Data collection}

Each country represented in the SIOPE DIPG Network is committed to delivering data to the SIOPE DIPG Registry and Imaging Repository. After subscription to the Network, the approved Network member receives a username and password to enter data into the Registry. Data collection covers both retrospective and prospective registration. Retrospective data will be collected from local hospitals, national registries and clinical trials. For prospective registration, Network members are encouraged to inform their patients about the existence of the SIOPE DIPG Registry followed by the informed consent procedure. In case of decline, the e-CRFs will be left blank, but a unique Registry number is created, which will only be used for epidemiologic studies. To describe data retrieval, as well as responsibility and ownership of the data, uniform international agreements for collaborative research purposes were created (see Legal aspects).

\section{Exhaustivity check and quality control of the data}

To ensure the reliability, validity, and completeness of the data [17], an appropriate program of Quality Control was implemented (Supplementary Fig. 1). Quality Control of data is an integral part of the project and takes place at all stages: before, during and after data entry.

\section{Data storage and safety}

Based on the e-CRF's, an optimized relational database was constructed. The database along with the web application is hosted on a dedicated server where the web application is the single point of contact with the database. All end-user connections use the secure HTTP (HTTPS) protocol to ensure protection of the privacy and integrity of the exchanged data. The server is placed within a Virtual Private LAN protected by a dedicated firewall ring. For server maintenance purposes direct access to the server is only possible through a restricted virtual private network (VPN) connection. The DIPG Registry is built on a generic framework in which presentation, logic and data layer are separated. The framework was designed with several active protection features to prevent unsolicited use of the application such as user/role/session validation, the use of antiforgery 
tokens and brute force protection. To ensure data safety, database input controls and extensive audit trailing are used. Every action within the system and the database is logged. The server, application and database are monitored $24 \mathrm{~h} / 7$ days and backups are made and stored daily on a different server in order to provide a disaster recovery scenario. The SIOPE DIPG Registry framework herewith provides a stable, secure and generic basis in any of its products. A penetration test (black box approach) was performed to validate the effectiveness of the (visible) security implemented on the SIOPE DIPG Registry and Imaging Repository. This test will be repeated on a regular basis.

\section{Legal aspects}

The daily and financial management, and hosting of the SIOPE DIPG Registry is carried out by the Dutch Childhood Oncology Group (DCOG), a National Paediatric Haematology-Oncology Society (NaPHOS) member of SIOPE. DCOG is mandated by the Executive Committee of the DIPG Network to act as a legal entity on its behalf in matters concerning the DIPG Registry, by a letter of mandate.

The construction of a collaborative research infrastructure, with geographical differences in health care structures and legislation faces considerable challenges. Experts in the field of sensitive data transfer and access rights have been consulted to certify issues concerning data anonymization, -collection, and -safety. To meet multinational standards, two legal documents have been drafted, abiding to EU law and taking into account SIOPE DIPG Network members' national laws. The first contains the SIOPE DIPG Network Bylaws (Supplementary material 3), that describe the mission, aims, core values and structure of the SIOPE DIPG Network as well as terms and conditions for submitting, reviewing and approving proposals for research projects using data from the SIOPE DIPG Registry. Furthermore, the Bylaws provide a Scientific Advisory Agreement for consultation of experts outside the SIOPE DIPG Network, such as specialised neuroradiologist for central radiology review. Second is the SIOPE DIPG Registry and Imaging Repository Regulatory Document (Supplementary material 4), describing the terms and conditions for management, maintenance of and access to the DIPG Registry and Imaging Repository.

\section{Use of data}

For strategic decisions concerning novel collaborative clinical and biological research projects in the field of DIPG, NCs meet or consult several times a year. In this way the SIOPE DIPG Network itself is responsible for the optimal use of obtained data. Data from the SIOPE DIPG Registry and Imaging Repository are available to researchers for collaborative, interdisciplinary, and translational studies. For use of the data from the Registry, the researcher must be a member of the SIOPE DIPG Network. The availability of data to the researcher is conditional to obtained approval from the Executive Committee, after submission of a project proposal, and permits and licenses required by the researcher's national law. The Executive Committee may set additional conditions to a specific project and stipulates the general terms and conditions with regard to receipt and use of data. Subsequently, only requested, relevant data are selected from the DIPG Registry and made available to the researcher. The researcher owns results of a research project, including the intellectual property rights thereto. Publication of results generated with data from the SIOPE DIPG Registry requires to comply with rules concerning authorship, as defined by the International Committee of Medical Journal Editors (ICMJE). Each year, the Executive Committee sends a report to the members of the SIOPE DIPG Network on the number of approved, performed and rejected projects.

\section{Results}

\section{International collaboration in DIPG research}

Since its inception in 2011, the SIOPE DIPG Network has expanded each year. Currently, 27 countries (Austria, Belgium, Croatia, Czech Republic, Denmark, Finland, France, Germany, Greece, Hungary, Iceland, Ireland, Italy, Lithuania, Norway, Poland, Portugal, Slovakia, Slovenia, Spain, Sweden, Switzerland, The Netherlands, United Kingdom, Turkey, Russia, and Mexico; Supplementary Fig. 2) have committed to the SIOPE DIPG Network and Registry. There is also a close collaboration with the International DIPG Registry, which represents the collaborative efforts of physicians and researchers from North America, Canada, Australia and New Zealand (Supplementary Fig. 2). To coordinate similar data collection, there are frequent telephone conferences and annual working visits between the SIOPE DIPG Network chair, the SIOPE DIPG Registry coordinator and International DIPG Registry team stationed at the Clinical Management and Research Support Core (CMRSC) at Cincinnati Children's Hospital Medical Center. Both DIPG registries are financially supported by The DIPG Collaborative, a collection of more than 20 parent foundations with the common interest of promoting and funding research into DIPG. 


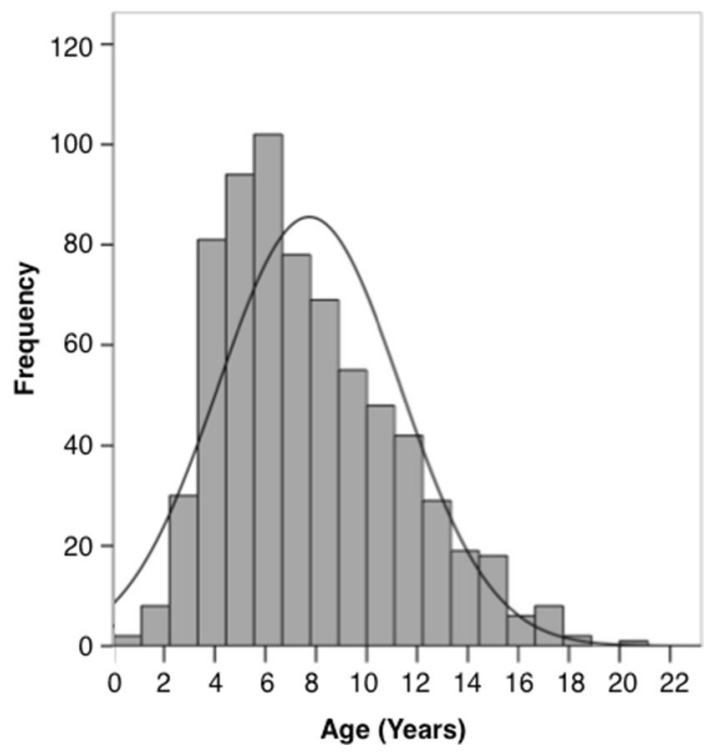

Fig. 3 Histogram showing the age distribution of the total cohort

\section{SIOPE DIPG registry and imaging repository}

Currently, as a prerequisite to start prospective patient inclusion in the SIOPE DIPG Registry, members of the SIOPE DIPG Network are in the process of Medical Ethical Committee and IRB review, with some countries already including patient data upon approval. As of April 2016, six countries have submitted retrospective data of 694 patients to the SIOPE DIPG Registry and Imaging Repository. Data were retrieved from three national registries, two local hospitals, and one clinical trial. Figure 3 shows the age distribution of patients included in the SIOPE DIPG Registry, with a median age of 7 years (standard deviation $(\mathrm{SD}) \pm 3.5)$. Table 1 shows the patient characteristics, clinical, radiological and biological disease characteristics, and treatment details of the total cohort. For 94 patients, tumor material was available for genetic analysis. Results are shown in Table 2. The median progression free survival, defined as time from diagnosis to clinical signs of disease progression (i.e., increase of symptoms or new symptoms) and/or radiological tumor progression on MRI, was 6.0 months (95\% Confidence Interval (CI) 5.6-6.4 months). The median OS, defined as time from diagnosis to death, was 11.0 months (95\% CI 10.5-11.5 months). PFS and OS are both plotted in Fig. 4a. Figure 4b, c show the PFS and OS stratified by mutational status. Figure $4 d$, finally, shows the distribution of time from progression to death (median 4 months). Ten percent of patients were alive at 2 years post diagnosis. At 5 years post diagnosis only two percent were alive. No disease-free survival was observed.

\section{Discussion}

A first step is made to improve the infrastructure of research into DIPG. This was done by (1) the establishment of the SIOPE DIPG Network, and (2) the development and initiation of the SIOPE DIPG Registry and Imaging Repository. This initiative, enabling collaborative research, is seen as major first step towards improving care and (ultimately) cure for children with DIPG.

Collaboration is pursued to overcome the factors hampering research into DIPG. This paper is the first to publish pooled patient data of almost 700 DIPG patients collected from national registries, local hospitals and clinical trials. To date, published patient data are largely from phase I/ II trials, which cover only a small percentage of the actual population diagnosed with DIPG. This possibly results in publication/selection bias. Future registration of all DIPG patients, both in- and outside trials, will give the opportunity to analyze 'real-life' DIPG patient data resulting in better description of incidence, characteristics and survival of DIPG patients. Also, it will generate a representative reference cohort, which may be used as historical control in any future study. With the SIOPE DIPG Network and SIOPE DIPG Registry/Imaging Repository, an infrastructure has been created that allows for research transparency, international collaboration and the elimination of duplication of research efforts. Already two international studies were published by the SIOPE DIPG Network, concerning palliative care and end-of-life decisions [18] and steroid use [19] in DIPG patients. The first large-scale international study including all patients registered in the SIOPE DIPG Registry and International Registry, with an estimated total of $>1000$ DIPG cases, is currently being conducted. This study will evaluate the characteristics of long-term surviving patients in comparison to the total group of patients.

The preliminary patient data of the 694 patients currently included in the SIOPE DIPG Registry, shows an equal gender distribution, rapid onset of symptoms prediagnosis $(86 \%<12$ weeks of which $66 \%$ within 6 weeks), a clinical presentation including cranial nerve palsy in the majority ( $85 \%$ ) of patients, and two-third of patients showing gadolinium contrast enhancement on the diagnostic MRI, of which $57 \%$ (39\% of the total cohort) showed partial ring-like enhancement suggestive for necrosis. At time of diagnosis, only $1 \%$ of the diagnostic MRIs showed metastasis in the brain, and $2 \%$ in the spine. Eighteen percent of patients present with hydrocephalus. Biopsy was performed in one-third of the patients, showing a range of WHO grades. From the 94 patients in whom histone mutational status was determined, two-third harbored a H3F3A mutation, versus $21 \%$ of patients harboring a H1H3B mutation, and $16 \%$ were classified as wild-type. This distribution, as well as the observed difference in survival in favor 
Table 1 Demographics, disease characteristics and treatment data of the total cohort $(n=694)$

\begin{tabular}{|c|c|c|c|}
\hline Category & Variable & $\mathrm{n}$ & $\begin{array}{l}\text { Valid } \\
(\%)\end{array}$ \\
\hline Total & & 694 & \\
\hline \multirow[t]{6}{*}{ Country } & Germany & $312 / 694$ & 45 \\
\hline & Netherlands & $132 / 694$ & 19 \\
\hline & France & $118 / 694$ & 17 \\
\hline & Italy & $79 / 694$ & 11 \\
\hline & United Kingdom & $45 / 694$ & 7 \\
\hline & Croatia & $8 / 694$ & 1 \\
\hline \multirow[t]{2}{*}{ Gender } & Female & $359 / 694$ & 52 \\
\hline & Male & $335 / 694$ & 48 \\
\hline Age & (mean, SD) & 7.7 & \pm 3.5 \\
\hline \multirow[t]{4}{*}{ Symptom duration } & $<6$ weeks & $413 / 627$ & 66 \\
\hline & 6-12 weeks & $127 / 627$ & 20 \\
\hline & 13-24 weeks & $47 / 627$ & 8 \\
\hline & $>24$ weeks & $40 / 627$ & 6 \\
\hline \multirow[t]{2}{*}{ Cranial nerve palsy } & Yes & $484 / 568$ & 85 \\
\hline & No & $84 / 568$ & 15 \\
\hline \multirow[t]{2}{*}{ Pyramidal signs } & Yes & $270 / 562$ & 48 \\
\hline & No & $292 / 562$ & 52 \\
\hline \multirow[t]{2}{*}{ Cerebellar signs } & Yes & $338 / 562$ & 60 \\
\hline & No & $224 / 562$ & 40 \\
\hline \multirow[t]{3}{*}{ T1-weighted } & Hypo-intense & $422 / 439$ & 96 \\
\hline & Iso-intense & $16 / 439$ & 4 \\
\hline & Hyper-intense & $1 / 439$ & 0 \\
\hline \multirow[t]{3}{*}{ T2-weighted } & Hypo-intense & $5 / 465$ & 1 \\
\hline & Iso-intense & $2 / 465$ & 0 \\
\hline & Hyper-intense & $458 / 465$ & 99 \\
\hline \multirow[t]{2}{*}{ Pontine involvement } & $<50 \%$ & $3 / 550$ & 0 \\
\hline & $>50 \%$ & $547 / 550$ & 100 \\
\hline \multirow[t]{3}{*}{ Tumor size } & Anterior-posterior $\varnothing$ in mm (mean, SD) & 36 & \pm 7 \\
\hline & Transverse $\varnothing$ in mm (mean, SD) & 43 & \pm 8 \\
\hline & Cranial-caudal $\varnothing$ in mm (mean, SD) & 42 & \pm 9 \\
\hline \multirow[t]{2}{*}{ Enhancement } & Yes & $336 / 516$ & 65 \\
\hline & No & $180 / 516$ & 35 \\
\hline \multirow[t]{2}{*}{ Ring-enhancement } & Yes & $191 / 491$ & 39 \\
\hline & No & $300 / 491$ & 61 \\
\hline \multirow[t]{2}{*}{ Margin } & Ill-defined & $363 / 481$ & 76 \\
\hline & Well-defined & $118 / 481$ & 24 \\
\hline \multirow[t]{2}{*}{ Extension } & Yes & $493 / 549$ & 90 \\
\hline & No & $56 / 549$ & 10 \\
\hline \multirow[t]{2}{*}{ Metastasis brain } & Yes & $7 / 547$ & 1 \\
\hline & No & $540 / 547$ & 99 \\
\hline \multirow[t]{2}{*}{ Metastasis spine } & Yes & $8 / 420$ & 2 \\
\hline & No & $412 / 420$ & 98 \\
\hline \multirow[t]{2}{*}{ Hemorrhage } & Yes & $60 / 458$ & 13 \\
\hline & No & $398 / 458$ & 87 \\
\hline \multirow[t]{2}{*}{ Necrosis } & Yes & $191 / 473$ & 40 \\
\hline & No & $282 / 473$ & 60 \\
\hline \multirow[t]{2}{*}{ Hydrocephalus } & Yes & $89 / 505$ & 18 \\
\hline & No & $416 / 505$ & 82 \\
\hline Radiation & Yes & $650 / 691$ & 94 \\
\hline
\end{tabular}


Table 1 (continued)

\begin{tabular}{|c|c|c|c|}
\hline Category & Variable & $\mathrm{n}$ & $\begin{array}{l}\text { Valid } \\
(\%)\end{array}$ \\
\hline & No & $41 / 691$ & 6 \\
\hline \multirow[t]{13}{*}{ Chemotherapy at diagnosis } & Yes & $498 / 689$ & 72 \\
\hline & $*$ Oral & $252 / 495$ & 51 \\
\hline & $* I V$ & $230 / 495$ & 46 \\
\hline & $*$ Both & $13 / 495$ & 3 \\
\hline & *Cytotoxic & $323 / 495$ & 65 \\
\hline & $*$ Targeted & $129 / 495$ & 26 \\
\hline & $*$ Both & $43 / 495$ & 9 \\
\hline & *EGFR & $111 / 495$ & 22 \\
\hline & *mTOR / PI3K & $15 / 495$ & 3 \\
\hline & *EGFR/mTOR & $1 / 495$ & 0 \\
\hline & *HDAC inhibitor & $37 / 495$ & 8 \\
\hline & $*$ Other & $331 / 495$ & 67 \\
\hline & No & $191 / 689$ & 28 \\
\hline \multirow[t]{2}{*}{ Chemotherapy at progressive disease } & Yes & $370 / 684$ & 54 \\
\hline & No & $314 / 684$ & 46 \\
\hline \multirow[t]{2}{*}{ Re-irradiation } & Yes & $61 / 694$ & 9 \\
\hline & No & $633 / 694$ & 91 \\
\hline \multirow[t]{2}{*}{ Hydrocephalus treatment } & Yes & $158 / 694$ & 23 \\
\hline & No & $536 / 694$ & 77 \\
\hline \multirow[t]{16}{*}{ Biopsy } & Yes & $260 / 694$ & 37 \\
\hline & *WHO Grade IV & $91 / 260$ & 35 \\
\hline & *Glioblastoma multiforme & $76 / 91$ & 84 \\
\hline & $* \mathrm{DIPG}^{\wedge}$ & $15 / 91$ & 16 \\
\hline & *WHO Grade III & $71 / 260$ & 27 \\
\hline & *Anaplastic astrocytoma & $61 / 71$ & 86 \\
\hline & *Anaplastic oligoastrocytoma & $8 / 71$ & 11 \\
\hline & *Anaplastic oligodendroglioma & $2 / 71$ & 3 \\
\hline & $*$ WHO Grade II & $38 / 260$ & 15 \\
\hline & *Diffuse astrocytoma & $20 / 38$ & 53 \\
\hline & *Low-grade astrocytoma n.o.s & $11 / 38$ & 29 \\
\hline & *Fibrillary astrocytoma & $4 / 38$ & 10 \\
\hline & $*$ Oligoastrocytoma & $2 / 38$ & 5 \\
\hline & *Oligodendroglioma & $1 / 38$ & 3 \\
\hline & *WHO Grade unknown & $60 / 260$ & 23 \\
\hline & No & $434 / 694$ & 63 \\
\hline \multirow[t]{7}{*}{ Autopsy } & Yes & $16 / 380$ & 4 \\
\hline & *WHO Grade IV & $12 / 16$ & 75 \\
\hline & *Glioblastoma multiforme & $12 / 12$ & 100 \\
\hline & *WHO Grade II-IV & $1 / 16$ & 6 \\
\hline & $*$ Astrocytoma & $1 / 1$ & 100 \\
\hline & *WHO Grade unknown & $3 / 16$ & 19 \\
\hline & No & $364 / 380$ & 96 \\
\hline
\end{tabular}

SD Standard Deviation, AP Anterior-posterior, WHO World Health Organization, *Following the 2016 WHO classification criteria [15]

of the $\mathrm{H} 1 \mathrm{H} 3 \mathrm{~B}$ mutational subgroup, is in line with international literature $[4,6,9,20]$. Almost all patients received radiotherapy, $9 \%$ received re-irradiation, and a sticking
$72 \%$ received chemotherapy, which is contradictory since there is no chemotherapeutic strategy yet, that has shown to be effective $[1,2]$. Autopsy was performed in only $4 \%$ 
Table 2 Genetic characteristics of patients with available tumor material $(\mathrm{n}=94)$

\begin{tabular}{llll}
\hline Category & Variable & $\mathrm{n}$ & VALID \% \\
\hline Total & & 94 & \\
Material type & Biopsy & $86 / 94$ & 92 \\
Histone mutations & Autopsy & $8 / 94$ & 8 \\
& H3F3A & $59 / 94$ & 63 \\
& H1H3B & $20 / 94$ & 21 \\
& H1H3C & $0 / 16$ & - \\
Additional mutations & H1H3I & $0 / 16$ & - \\
& Wild-type & $15 / 94$ & 16 \\
& ACVR1 & $9 / 45$ & 17 \\
& Wild-type & $45 / 54$ & 83 \\
& TP53 & $18 / 29$ & 62 \\
& Wild-type & $11 / 29$ & 38 \\
& ATM & $3 / 16$ & 19 \\
& Wild-type & $13 / 16$ & 81 \\
& PIK3CA & $5 / 30$ & 17 \\
& Wild-type & $25 / 30$ & 83 \\
& PIK3R1 & $3 / 15$ & 20 \\
& Wild-type & $12 / 15$ & 80 \\
& MET & $1 / 15$ & 7 \\
& Wild-type & $14 / 15$ & 93 \\
\hline
\end{tabular}

of patients. Currently, the majority of patients included in the SIOPE DIPG Registry are patients with a radiologically confirmed and centrally reviewed $\mathrm{T} 1$-weighted hypointense and T2-weighted hyperintense tumor with at least 50\% involvement of the pons (DIPG) [11]. The recent WHO re-classification, however, may imply that the inclusion criteria for the SIOPE DIPG Registry need to be adjusted to also include patients with non-pontine diffuse midline gliomas in the future.

Dependent on the extent to which biopsies and autopsies will be (re-)introduced for DIPG, data on biological characteristics will gradually increment in the Registry, which will increase the knowledge on DIPG etiology, pathogenesis, possible drug targets and the mechanisms that contribute to the observed resistance to treatment. Furthermore, big-data analysis of aggregated clinical, radiological and especially biological data facilitates the discovery of patterns that indicate patient subgroups, which enables consensus formation on classification, in-/exclusion and response criteria, and improves the quality and comparability of future trials. Moreover, joining forces within an international research-infrastructure will stimulate the initiation of, and active accrual in, international multicenter trials, with sufficient power to address the many unanswered research questions. This, together with the recent evolution of ideas concerning therapeutic strategies [21-24], should facilitate the identification and selection of novel tolerable and effective therapies.

Data collection in the Registry will have some (initial) limitations. Due to the former lack of local hospital- and national registrations, lack of specific ICD-codes ${ }^{1}$, and due to a presumed limited documentation of clinical, radiological and pathological data, retrospective data collection will very likely be incomplete. Based on data from the Dutch retrospective study [10], and included parties in the SIOPE DIPG Network (with a total number of about 600 million residents aged 0-19 years; April 2016) it is estimated that over 350 children are eligible for prospective registration in the SIOPE DIPG Registry each year. It is expected that annually about 200 patients $(60 \%)$ will be registered in the first years, and that this number will increase when the SIOPE DIPG Network expands, resulting in higher data completeness per country over time.

Recent publications in DIPG literature have shown that coupling genetic data to clinical data will become increasingly important to understand and/or predict the clinical behavior of the disease $[9,14]$. Therefore, as for now, data of the most common genetic aberrations are entered in the Registry via a 'Biopsy/Autopsy e-CRF'. A next step of the SIOPE DIPG Registry is to establish a (virtual) biobank of DIPG material, linked with the DIPG Genomics Repository at Progenetix (dipg.progenetix.org), a cancer genome database [25]. Ideally, the increased availability of DIPG tumor tissue will lead to generally available, representative, and possibly even patient subgroup-specific cell cultures and xenograft models, which enable thorough basic research and high-throughput screening of candidate therapies. Other future perspectives are to include questionnaires for Quality of Life research since research on this important subject is largely lacking, especially data on end-stage disease symptoms and the associated specific needs for palliative and end-of-life care [18]. The collection of conventional MR-imaging data in the Imaging Repository, will in the future be expanded to multimodality MR-imaging and other advanced imaging techniques such as PET. The data also might be useful for educational purposes (e-learning) in an aim to improve diagnostics of these tumors.

To conclude, with the collaborative efforts of professionals treating children with DIPG, patient/parent organizations, legal advisors, experts in the field of information technology and imaging experts, an international research-infrastructure was successfully set up, which led to the development and initiation of the SIOPE DIPG Registry. With already 694 patients registered, this Registry stimulates collaborative preclinical and clinical research efforts. The first study using data from both the SIOPE

\footnotetext{
1 World Health Organisation (WHO) International Classification of Diseases (ICD).
} 

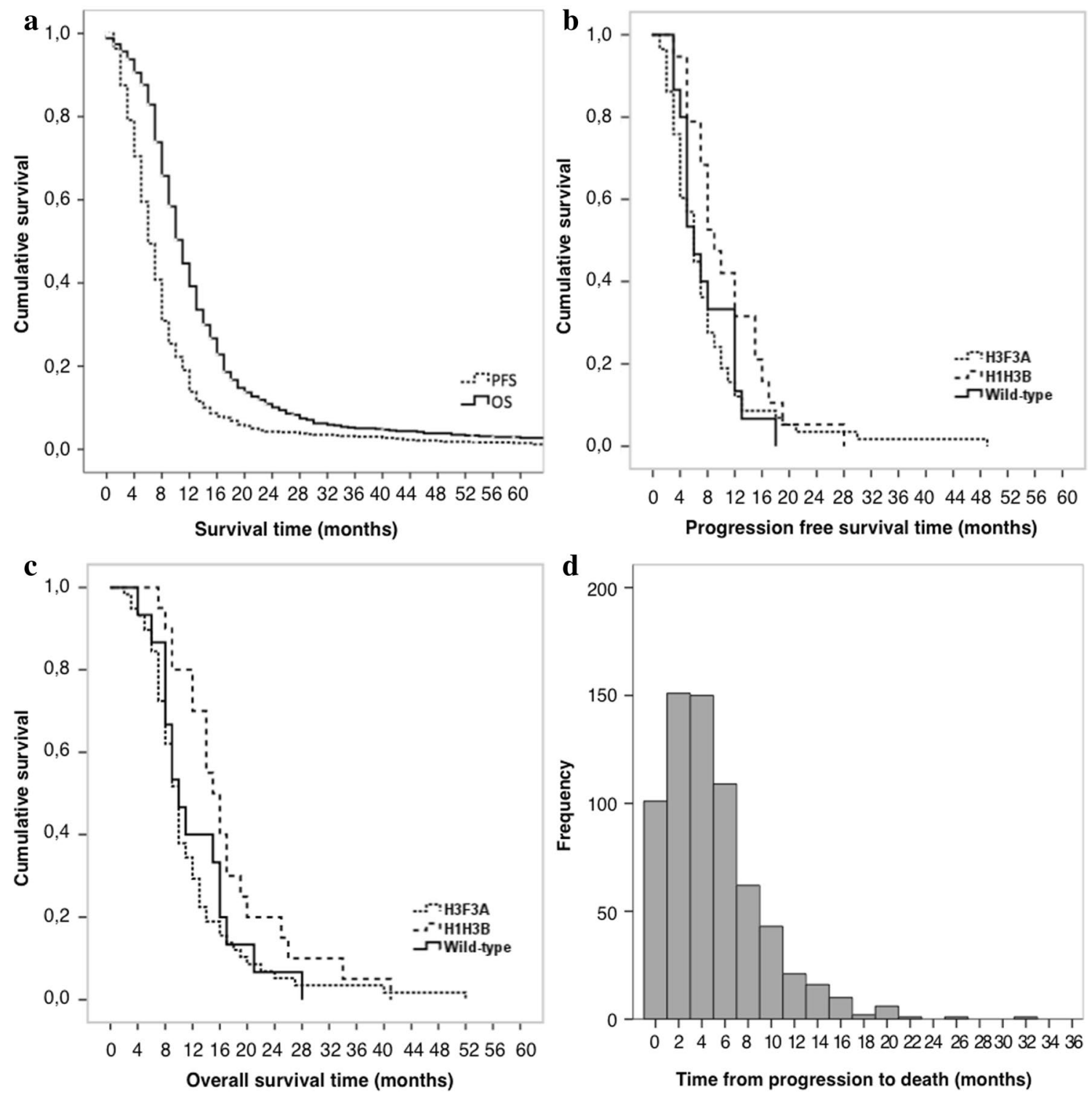

Fig. 4 Survival data. a Kaplan Meier estimates of progression free survival (PFS; $n=684$ ) and overall survival $(\mathrm{OS} ; \mathrm{n}=691)$. b Kaplan Meier estimates of progression free survival (PFS) stratified by mutational status (H3F3A $n=59, H 1 H 3 B n=20$, wild-type $n=15)$. c

and International Registry is already in its final stages. The existence of the International DIPG Registry, surveying similar data as the SIOPE DIPG Registry, allows for external cross-validation of data, generating robust data on the DIPG patient population. Big data analysis of the Registry's data will potentially lead to the discovery of patterns that pave the way to the identification of effective therapies towards a cure for patients suffering from DIPG.

The methodology used for the SIOPE DIPG Registry will, most likely, be easily translatable to other pediatric cancer registries, as almost all of these are orphan diseases that could benefit from international registration and collaboration in research.
Kaplan Meier estimates of overall survival (OS) stratified by mutational status (H3F3A $n=59$, H1H3B $n=20$, wild-type $n=15)$. d Histogram showing the distribution of time from progression to death

Acknowledgements We thank all the parents of children who have suffered from DIPG for their valuable contribution to this research, both in financing as in input for the SIOPE DIPG Registry: The Cure Starts Now Foundation, Hope for Caroline Foundation, Julian Boivin Courage for Cures Foundation, Abbie's Army, Michael Mosier Defeat DIPG Foundation, Brooke Healey Foundation, Reflections of Grace Foundation, The Cure Starts Now Australia, Soar With Grace Foundation, Jeffrey Thomas Hayden Foundation, Cure Brain Cancer Foundation, The Jones Family Foundation, Musella Foundation for Brain Tumor Research and Information, The Pray, Hope Believe Foundation, Benny's World, Smiles for Sophie Forever, Aiden's Avengers, Love Chloe Foundation, A Cure From Caleb Society, Operation Grace White Foundation, Ryan's Hope, American Childhood Cancer Organization, Juliana Rose Donnelly Trust, Joshua's Wish, The Ellie Kavalieros DIPG Research Fund, Voices Against Brain Cancer, Wayland's Warriors, Sheila Jones \& Friends and The DIPG Collaborative. 
Special thanks go out to KPMG for the pro bono support of the project and for the validation of the design and security of the SIOPE DIPG Registry and Imaging Repository. SVvZ and EH are financially supported by the Semmy Foundation (Stichting Semmy), MM and VB are financially supported by Associazione Bianca Garavaglia Onlus, Il fondo di Giò, DVU is financially supported by VUmc-CCA*Children.

\section{Compliance with ethical standards}

Conflict of interest The authors declare that they have no conflict of interest.

Informed consent If required, a SIOPE Network member informed parents (and patients), after which he/she provided the Patient Information Form and requested for informed consent.

Research involving human participants All data in the SIOPE DIPG Registry has been retrieved from the participating hospital upon local Medical Ethical Committee and IRB approval. The SIOPE DIPG Registry project is being performed in accordance with the ethical standards as laid down in the 1964 Declaration of Helsinki and its later amendments or comparable ethical standards.

Open Access This article is distributed under the terms of the Creative Commons Attribution 4.0 International License (http:// creativecommons.org/licenses/by/4.0/), which permits unrestricted use, distribution, and reproduction in any medium, provided you give appropriate credit to the original author(s) and the source, provide a link to the Creative Commons license, and indicate if changes were made.

\section{References}

1. Hargrave D, Bartels U, Bouffet E (2006) Diffuse brainstem glioma in children: critical review of clinical trials. Lancet Oncol 7:241-248. doi:10.1016/S1470-2045(06)70615-5

2. Jansen MHA, van Vuurden DG, Vandertop WP, Kaspers GJL (2012) Diffuse intrinsic pontine gliomas: A systematic update on clinical trials and biology. Cancer Treat Rev 38:27-35. doi:10.1016/j.ctrv.2011.06.007

3. Grill J, Puget S, Andreiuolo F, et al (2012) Critical oncogenic mutations in newly diagnosed pediatric diffuse intrinsic pontine glioma. Pediatr Blood Cancer 58:489-491. doi:10.1002/pbc.24060

4. Khuong-Quang D-AA, Buczkowicz P, Rakopoulos P et al (2012) K27M mutation in histone H3.3 defines clinically and biologically distinct subgroups of pediatric diffuse intrinsic pontine gliomas. Acta Neuropathol 124:439-447. doi:10.1007/ s00401-012-0998-0

5. Fontebasso AM, Papillon-Cavanagh S, Schwartzentruber J et al (2014) Recurrent somatic mutations in ACVR1 in pediatric midline high-grade astrocytoma. Nat Genet 46:462-466. doi:10.1038/ng.2950

6. Wu G, Diaz AK, Paugh BS et al (2014) The genomic landscape of diffuse intrinsic pontine glioma and pediatric non-brainstem high-grade glioma. Nat Genet 46:444-450. doi:10.1038/ng.2938

7. Taylor KR, Mackay A, Truffaux N et al (2014) Recurrent activating ACVR1 mutations in diffuse intrinsic pontine glioma. Nat Genet 46:457-461. doi:10.1038/ng.2925

8. Buczkowicz P, Hoeman C, Rakopoulos P et al (2014): Genomic analysis of diffuse intrinsic pontine gliomas identifies three molecular subgroups and recurrent activating ACVR1 mutations. Nat Genet 46:451-456. doi:10.1038/ng.2936
9. Castel D, Philippe C, Calmon R et al (2015) Histone H3F3A and HIST1H3B K27M mutations define two subgroups of diffuse intrinsic pontine gliomas with different prognosis and phenotypes. Acta Neuropathol 130:815-827. doi:10.1007/ s00401-015-1478-0

10. Veldhuijzen van Zanten SEM, Jansen MHA, Sanchez Aliaga $E$ et al (2015) A twenty-year review of diagnosing and treating children with diffuse intrinsic pontine glioma in The Netherlands. Expert Rev Anticancer Ther 15:157-164. doi:10.1586/14 737140.2015.974563

11. Barkovich AJ, Krischer J, Kun LE et al (1990) Brain stem gliomas: a classification system based on magnetic resonance imaging. Pediatr Neurosurg 16:73-83

12. Warren KE (2012) Diffuse intrinsic pontine glioma: poised for progress. Front Oncol 2:205. doi:10.3389/fonc.2012.00205

13. Albright AL, Packer RJ, Zimmerman R et al (1993) Magnetic resonance scans should replace biopsies for the diagnosis of diffuse brain stem gliomas: a report from the Children's Cancer Group. Neurosurgery 33:1026-1030. doi:10.1227/00006123-199312000-00010

14. Jansen MHA, Veldhuijzen van Zanten SEM, Heymans MW et al (2016) Commentary on "Histone H3F3A and HIST1H3B K27M mutations define two subgroups of diffuse intrinsic pontine gliomas with different prognosis and phenotypes". Acta Neuropathol 131:793-794. doi:10.1007/s00401-016-1567-8

15. Louis DN, Perry A, Reifenberger G et al (2016) The 2016 World Health Organization classification of tumors of the central nervous system: a summary. Acta Neuropathol 131:803-820. doi:10.1007/s00401-016-1545-1

16. John EM, Hopper JL, Beck JC et al (2004) The Breast Cancer Family Registry: an infrastructure for cooperative multinational, interdisciplinary and translational studies of the genetic epidemiology of breast cancer. Breast Cancer Res 6:R375-R389. doi:10.1186/bcr801

17. Arts DGT, De Keizer NF, Scheffer GJ (2002) Defining and improving data quality in medical registries: a literature review, case study, and generic framework. J Am Med Inform Assoc 9:600-611. doi:10.1197/jamia.M1087

18. Veldhuijzen van Zanten SEM, van Meerwijk CLLI, Jansen MHA et al (2015) Palliative and end-of-life care for children with diffuse intrinsic pontine glioma: results from a London cohort study and international survey. Neuro Oncol 18:582-588 doi:10.1093/ neuonc/nov250

19. Veldhuijzen van Zanten SEM, Cruz O, Kaspers GJL et al (2016) State of affairs in use of steroids in diffuse intrinsic pontine glioma: an international survey and a review of the literature. $\mathbf{J}$ Neurooncol 128:387-394. doi:10.1093/neuonc/nov250

20. Wu G, Broniscer A, McEachron TA, et al (2012) Somatic histone $\mathrm{H} 3$ alterations in pediatric diffuse intrinsic pontine gliomas and non-brainstem glioblastomas. Nat Genet 44:251-253. doi:10.1038/ng.1102

21. Saletta F, Wadham C, Ziegler DS et al (2014) Molecular profiling of childhood cancer: biomarkers and novel therapies. BBA Clin 1:59-77. doi:10.1016/j.bbacli.2014.06.003

22. Buczkowicz P, Bartels U, Bouffet E et al (2014) Histopathological spectrum of paediatric diffuse intrinsic pontine glioma: diagnostic and therapeutic implications. Acta Neuropathol 128:57381. doi:10.1007/s00401-014-1319-6

23. Grasso CS, Tang Y, Truffaux N et al (2015) Functionally defined therapeutic targets in diffuse intrinsic pontine glioma. Nat Med 21:555-559. doi:10.1038/nm.3855

24. Morales La Madrid A, Hashizume R, Kieran MW (2015) Future clinical trials in DIPG: bringing epigenetics to the clinic. Front. Oncol 5:1-5. doi:10.3389/fonc.2015.00148

25. Baudis M, Cleary ML (2001) Progenetix.net: an online repository for molecular cytogenetic aberration data. Bioinformatics $17: 1228-1229$ 